\title{
Cryogenic high-Q microwave resonators for stable oscillators
}

\author{
S. Vitusevich, M. Winter, and N. Klein
}

\begin{abstract}
Cryogenic microwave resonators have a strong potential as frequency stabilising elements for oscillators to be used in advanced radar systems and high-bitrate microwave communication links. Depending on frequency, either 2D planar HTS-resonators, HTS-shielded sapphire $\mathbf{T E}_{011}$ resonators or cryogenic sapphire whispering-gallery mode resonators represent the best compromise between resonator quality factor and size. We have built and tested an all-cryogenic oscillator based on a WG-resonator at $f=23 \mathrm{GHz}$. Phase noise measurements indicate values superior to quartz stabilized oscillators. A two-step electric frequency tuning consisting of an integrated varactor phase shifter and a dielectric plunger moved by a piezomechanical transducer is introduced to compensate frequency drifting with temperature. For further improvement of long-time frequency stability we have developed rutilesapphire composite dielectric resonators. Due to the opposite sign of the temperature slope of the dielectric constant of sapphire and rutile a turning point appears in the temperature dependence of the resonance frequency. Employing a moderate temperature stabilization as good as a few millikelvin around the turning point at $T=78 \mathrm{~K}$, we have demonstrated a long time frequency stability at least as good as for oven controlled quartz oscillators.
\end{abstract}

Index Terms-low phase noise oscillators, dielectric resonators, cryocoolers, dielectric losses

\section{INTRODUCTION}

$\mathrm{W}$ hispering-gallery modes are modes with high azimuthal mode number which can exist if boundary conditions with (nearly) circular symmetry are present. The name originates from sound waves being totally reflected from the cylindrically shaped wall inside the socalled "whispering gallery" of St.Paul's cathedral in London. For electromagnetic waves total reflection occurs at shallow incident angles at the transition from a medium with high to a medium with low refractive index. Due to this phenomena whispering-gallery modes in dielectric cylinders machined from high-purity sapphire single crystals provide the highest unloaded quality factors above liquid helium temperature, which are close to that of superconducting

Manuscript received September 18, 2000. This work was supported in part by Bosch Telecom in the framework of a project funded by the German ministry of research an education. Additional funding has been provided by the European Commission in the framework of the EU projects "DiHiMiCo" and "FOAMS".

The authors are at Forschungszentrum Jülich GmbH, Institute of Thin Film and Ion Technology, D-52425 Juclich, Germany, corresponding cmail: n.klein@fz-juelich.de niobium cavities at temperatures below about $50 \mathrm{~K}$ [1]. This is due to the low values of the loss tangent of sapphire which drops almost proportional to $T^{5}$ from $7 \cdot 10^{-6}$ at room temperature to $6 \cdot 10^{-8}$ at $77 \mathrm{~K}$. The permittivity of sapphire $\left(\varepsilon_{\mathrm{r}, \mathrm{a}, \mathrm{b}}=9.4, \varepsilon_{\mathrm{i}, \mathrm{c}}=11.4\right)$ is high enough to allow for total reflection at moderate incident angles $\alpha=\arcsin \left(1 / \varepsilon_{\mathrm{r}}{ }^{1 / 2}\right) \approx$ $19^{0}$ (with respect to the surface normal). This means, that very high quality factors can be expected for moderate values of the azimuthal mode number $m$, which is a measure for the number of reflections per circumference.
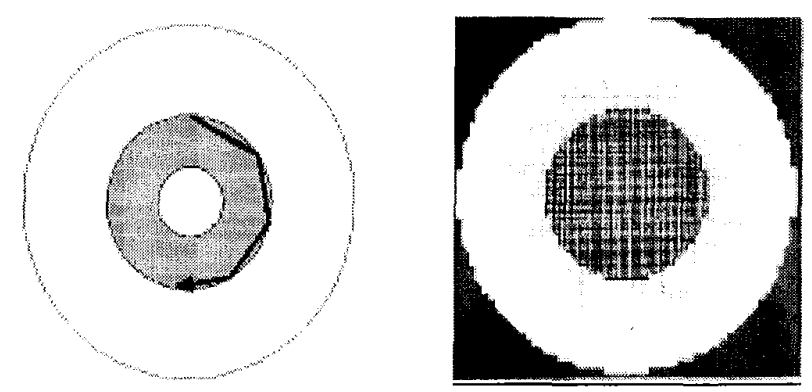

Fig. I Basic concept and numerically calculated electric ficld energy distribution outside the dielectric cylinder (light grey area) of a WGresonance with $m=7$.

In addition to very high $Q$ values at temperatures accessible with low-power cryocoolers, the strong field confinement inside the sapphire cylinder and the high mechanical stability of sapphire make sapphire whispering-gallery (WG) mode resonators attractive to be used as frequency stabilizing elements in microwave circuits.

\section{ALL CRYOGENIC K-BAND OSCILLATOR}

For high-data-rate up/down or crosslink for future multimedia satellites at Ka-band frequencies the effective date rate may be increased using higher order phase modulation schemes. Usually, the bit sequences are translated into numbers of the basis of $2^{n}, n=1,2,3 \ldots$ Each of the $2^{n}$ digits of a number corresponds to one of the $2^{n}$ allocated phase shift values between $0^{\circ}$ and $360^{\circ}$. During data transmission, phase flips of $k$ times $360^{\circ} / 2^{n}\left(k=1,2, \ldots, 2^{n}\right)$ occur leading to sidebands at offset frequencies of $\left(t_{\text {swikh }} 2^{n}\right)^{-1}$, where $t_{s w i c h}$ is the switching time of the modulator /demodulator. Thus for increasing $n$ the sidebands move closer to the carrier. The maximum number of $n$ is limited by 
bit errors due to the oscillator phase noise which increases strongly with decreasing frequency offset from the carrier.

As the most simple oscillator approach, a low $1 / f$ - noise microwave amplifier is feedbacked by a two-port microwave resonator. If the total phase shift around the oscillator loop is an integer of $2 \pi$ and the total loop amplification (equal to amplifier gain minus insertion loss of resonator and other components) exceeds unity, this device oscillates at one of the resonant frequency of the resonator. In addition, phase noise of the amplifier is translated into oscillator phase noise according to the Leeson model [2]:

$$
L_{a x c}=10 \cdot \log \left[1+\frac{f_{0}^{2}}{4 Q_{L}{ }^{2} f_{m}^{2}}\right]+10 \cdot \log \left[\frac{\alpha}{f_{m}}+\frac{G F k T}{P}\right]
$$

The second term in Eq. 1 represents the amplifier noise consisting of white noise determined by the amplifier gain $G$ its noise figure $F$, its physical temperature $T$ and its output power $P$ and of $1 / f$ noise leading to a fluctuation amplitude $\alpha d f_{m}$ at a frequency distance $\pm f_{m}$ from the carrier frequency $f_{0}$. The first term in Eq. 1 represents the resonator noise amplification factor which is $3 \mathrm{~dB}$ for $f_{m}=1 / 2 \Delta f_{l / 2}\left(\Delta f_{l / 2}=\right.$ $\left.f_{0} / Q_{L}\right)$ and increases strongly for lower offset frequencies According to Eq. 1 the oscillator phase noise exhibits a $1 / f_{m}{ }^{3}$ dependence and decreases strongly upon increasing the unloaded quality factor $Q_{L}$ of the resonator.

We have developed an all-cyrogenic K-band oscillator for operation at $f=23 \mathrm{GHz}$ with a three-step mechanical and electrical frequency tuning (Fig. 2). The parts of this hybrid oscillator are the following:

- A whispering gallery mode resonator with a mechanical tuning range of $60 \mathrm{MHz}$ and a piezomechanical fine tuning range of $50 \mathrm{kHz}$ (Fig. 3). The unloaded quality factor was found to be above $2 \cdot 10^{6}$ at $77 \mathrm{~K}$ over the entire tuning range. Tuning is provided by changing the physical distance between the sapphire resonator and a sapphire tuning disk.

- A cryogenic two-stage HEMT amplifier including a semiconductor varactor phase shifter and a $10 \mathrm{~dB}$ output coupler.

- A high-temperature superconducting two-pole dual-mode bandpass filter which avoids locking to any spurious mode

Two identical oscillators have been built in order to perform comparative phase-noise measurements. The oscillators were tested by operating both inside one vacuum dewar. Cooling to liquid nitrogen temperature was attained by mounting the oscillators on the copper bottom plate of the dewar. The bottom plate was cooled from the outside with liquid nitrogen. Temperature controlling was provided by employing a heat resistor and a temperature sensor for each oscillator, both connected to a Lake Shore temperature controller.

Phase noise measurements were performed employing a Hewlett-Packard phase noise measurement system operated in three different configurations (see also Fig. 4):

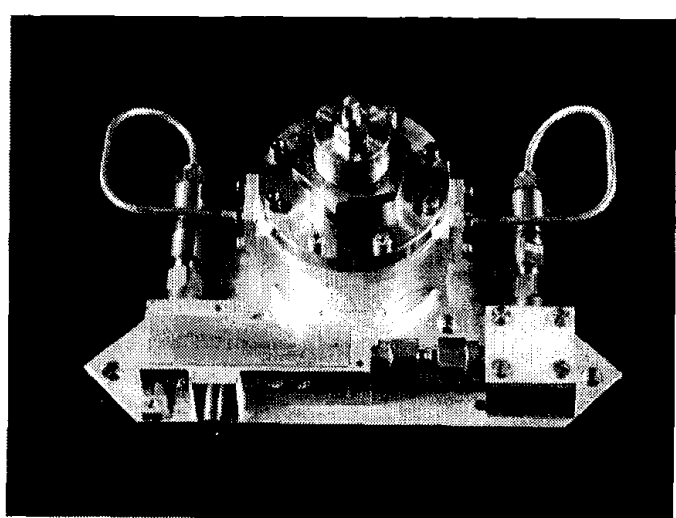

Fig.2 Photograph of the cryogenic $23 \mathrm{GHz}$ oscillator consisting of a whispering-gallery mode resonator (large circular metal housing), a 2stage HEMT-amplifier (rectangular housing) and a high-temperature superconducting bandpass filter for mode selection (square housing). The amplifier and the filter have been developed and fabricated by Bosch Telecom.

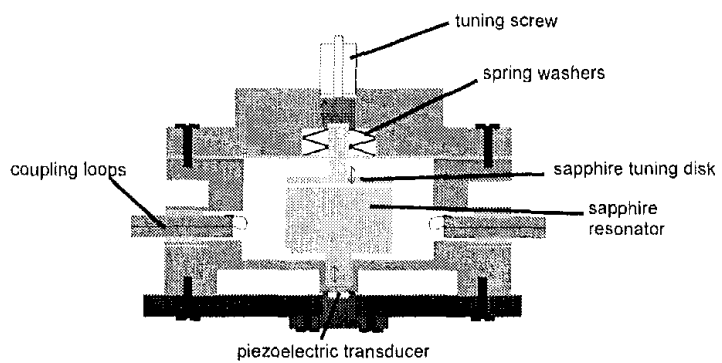

Fig.3 Tunable whispering-gallery mode resonator for $f=23 \mathrm{GHz}$ with mechanical / piezomechanical tuning range of $60 \mathrm{MHz} / 50 \mathrm{kHz}$.

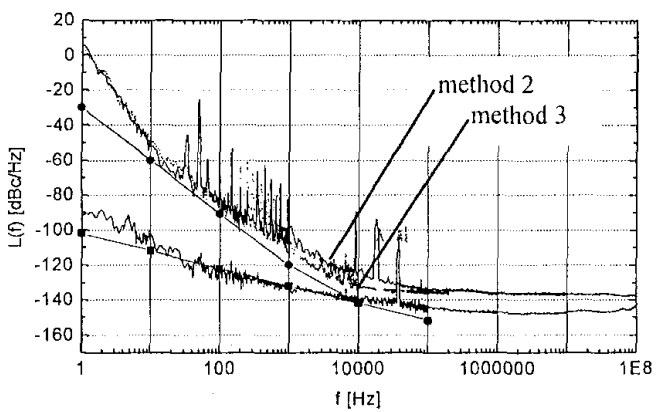

Fig.4 Measured phase noise of the amplifier (lower curve) and of the oscillator by method 2 and 3 . The squares represent a $1 / f$ fit to the amplifier noise, the dots represent the oscillator phase noise calculated from the fit to the amplifier phase noise according to Eq. 1 .

1. The amplifier phase noise is determined by splitting a 23 $\mathrm{GHz}$ CW signal from a HP Sweep synthesizer into two equal signals, passing one through the amplifier and the other through a mechanical phase shifter. The beat signal is used to extract the amplifier phase noise.

2. The signal of one oscillator is downconverted to $1.6 \mathrm{GHz}$ and the oscillator phase noise is measured against a lownoise HP quartz reference source. 
3. The signals of the two oscillators are directed to a mixer. Phase noise of the beat signal (typically around a few $\mathrm{MHz}$ ) is measured against the quartz reference. In contrast to method 2, the noise of the downconverter is omitted.

The loaded $Q$ value of the resonator was found to be $3 \cdot 10^{6}$ at $77 \mathrm{~K}$ for a resonator insertion loss of $-7 \mathrm{~dB}$. From the coupling coefficient the unloaded $Q$ was determined to be $6 \cdot 10^{6}$. From literature data [1] the loss tangent of sapphire is expected to be $\left(7.2 \cdot 10^{6}\right)^{-1}$ indicating that our resonator design allows for $Q$ values very close to the fundamental limits of quantum absorption by phonons.

Fig. 4 shows the measured phase noise. The amplifier phase noise was found to be nearly independent of temperature between room temperature and $77 \mathrm{~K}$. The measured values of amplifier phase noise indicate $1 / \mathrm{f}$ behaviour with an absolute value of $-132 \mathrm{dBc} / \mathrm{Hz}$ at $1 \mathrm{kHz}$ offset frequency. According to comparative measurements with commercial broad band amplifiers at the same frequency, this value is quite low.

The oscillator phase noise calculated from the amplifier phase noise according to Eq. 1 is $-120 \mathrm{dBc} / \mathrm{Hz}$ at $1 \mathrm{kHz}$ offset frequency. This is well below that of quartz reference based microwave oscillators at the same frequency. However, the oscillator phase noise measured by method $2 / 3$ are about $15 \mathrm{~dB} / 8 \mathrm{~dB}$ above (at $1 \mathrm{kHz}$ offset) the calculated values. This is due to the noise floor of the downconverter (method 2) and the reference source (methods 2 and 3 ).

The test of the oscillators on a cryocooler is still in progress. According to the results with the compensated WGresonator, we do not expect a significant influence of the cold head vibrations on the phase noise (see next chapter).

\section{TEMPERATURE COMPENSATED SAPPHIRE-RUTILE COMPOSITE RESONATOR}

One drawback of sapphire as material for dielectric resonators is the large temperature coefficient of the resonance frequency of about $30 \mathrm{ppm} / \mathrm{K}$ at $77 \mathrm{~K}$. Assuming a temperature stability of $\pm 50 \mathrm{mK}$ on a prospective cryoplatform in a satellite, the oscillator frequency would vary by $\pm 50 \mathrm{kHz}$. This is much higher than the halfwidth of $3.8 \mathrm{kHz}$ of the WG resonance indicating that tuning of the feedback circuit phase by a varactor is insufficient for electronic compensation of temperature fluctuations. For the current oscillator design the piezomechanical tuning of the WG-resonance frequency by $50 \mathrm{kHz}$ comes close to the required compensation range, but may not be very convenient e.g. due to creep effects in the piezoelectric material.

A very elegant approach to reduce the temperature coefficient of a sapphire WG resonator by orders of magnitude without a significant $Q$ reduction is the rutile compensation technique [3]. Utilizing the opposite sign of $\mathrm{d} \varepsilon_{r} / \mathrm{d} T$ of rutile and sapphire one can generate a turning point in the temperature dependence of the resonance frequency $f$, i.e. a temperature where $\mathrm{d} f / \mathrm{d} T=0$. Since the absolute value of the temperature coefficient of rutile is about a factor of 100 larger in comparison to sapphire and the loss tangent of rutile is in the $10^{-6}$ range [4], high quality factors close to that of pure sapphire can be expected for temperature compensated sapphire-rutile composite resonators [7].

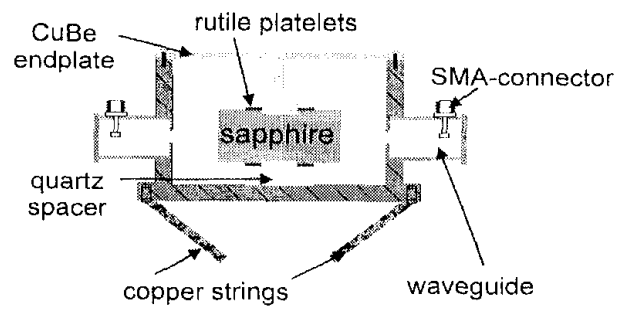

Fig. $5 X$-band rutile/sapphire composite whispering-gallery mode resonator with waveguide coupling (cross-section).

The design of the resonator is shown in Fig. 5 (for a more detailed discussion of the resonator design and experimental results see Ref. 5). Two rings of thin rutile platelets are pressed by a copper beryllium spring between quartz spacers and the central sapphire puck. According to numerical field

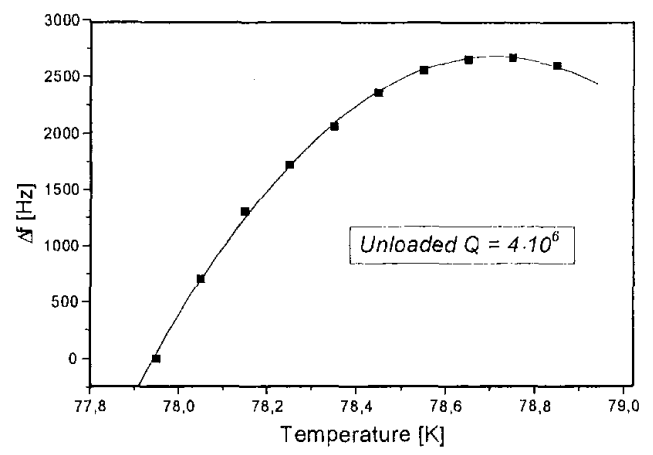

Fig. 6. Measured resonance frequency $(f-9.96310 \mathrm{GHz})$ versus temperature.

simulations with the computer code MAFIA [6] the electric filling factor of the rutile rings was found to be about $1 \%$.

In order to measure the long time frequency stability, we have assembled the resonator on the cold head of a pulse tube refrigerator using copper strings for mechanical isolation similar to the oscillator described before. The resonance frequency was determined from a fit to the Lorentzian shaped resonance curve measured with a vector network analyser $(V N A)$. As frequency reference we used an oven controlled quartz standard. Fig. 6 shows the measured temperature dependence of the resonance frequency. The recorded data points correspond to equilibrium values where the frequency is averaged over more than 1000 seconds. From a quadratic fit of the $f(T)$ data we calculated the relative frequency change with temperature around the turning point to be 
$-4.2 \cdot 10^{-13} / \mathrm{mK}^{2}$. This corresponds to a compensation curvature, $1 / f \partial^{2} f / \partial T^{2}$, of $-0.9 \mathrm{ppm} / \mathrm{K}^{2}$. According to this result, a frequency stability comparable to that of a rubidium standard should be possible if a temperature stability around a few millikelvin can be achieved.

The measured unloaded quality factor of $4 \cdot 10^{6}$ at the turning point is in agreement with the theoretical expectations from the loss tangent values of sapphire and rutile.

Fig. 7 shows the measured Allan variance of frequency and temperature calculated from the experimental data at the turning point over a period of more than 24 hours. For integration times $>1000 \mathrm{~s}$ the Allan variance of frequency corresponds to the specified frequency stability of our ovencontrolled quartz reference. This indicates, that our technique can provide long-time frequency performance at least as good as for quartz references. In order to measure the real performance, measurements against rubidium or even hydrogen standards still need to be performed.

At an integration time of about $250 \mathrm{~s}$ there one observes a

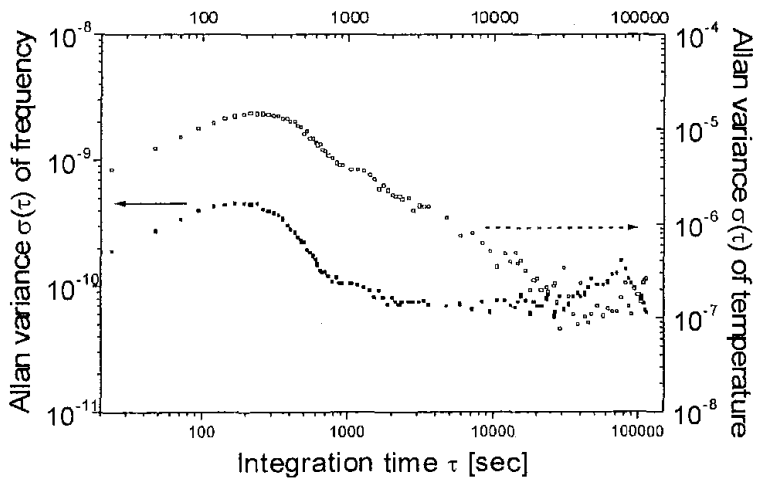

Fig. 7 Measured Allan variance of frequency and temperature from $\tau=10 \mathrm{~s}$ to $\tau=28 \mathrm{~h}$.

maximum both for the Allan variance of temperature and frequency. The maximum value corresponds to temperature fluctuation of $1.5 \mathrm{mK}$. Surprisingly, the measured frequency Allan variance maximum of about $5 \cdot 10^{-10}$ is a factor of 500 larger than expected from the equilibrium values at the turning point according to Fig. 6. Currently, we do not understand this discrepancy quantitatively. One possible explanation would be a temperature gradient between the sapphire puck and the rutile rings. In this case the effective linear frequency slope of each component, which is of the order of $50 \mathrm{ppm} / \mathrm{K}$, may generate much higher frequency fluctuations. In contrast to our results, both for superconducting niobium cavity and sapphire resonator standards operated a liquid helium temperatures frequency stability values below $10^{-15}$ for integration intervals below $1000 \mathrm{~s}$ were demonstrated $[8,9]$. We believe, that this discrepancy is mainly due to the absence of large "linear slopes" as for rutile and sapphire. Therefore, temperature gradient effects are expected to be negligible. In order to reduce the effect of temperature gradients in our setup, an improved thermal cavity design and possible operation at lower temperatures still attainable with low-power cryocoolers $(30-50 \mathrm{~K})$ will be investigated in the near future.

As an advantage with respect to atomic microwave frequency standards, the phase noise, i.e. the short time stability is expected to be much better. This is a generic advantage of passive electromagnetic resonator standards, which can be explained in general by the fact that photons do not interact with each other but electrons do.

In order to generate a low-phase noise signal with a longtime stability determined by the compensated rutile-sapphire resonator, we assembled a feedback oscillator similar to the one described before, but with a commercial amplifier at room temperature. The measured phase noise exhibits the expected $1 / f_{m}{ }^{3}$ dependence with an absolute value of -115 $\mathrm{dBc} / \mathrm{Hz}$ at $f_{m}=1 \mathrm{kHz}$. This is in very good agreement with the measured amplifier phase noise and resonator $Q_{l}$ according to Eq. 1. As a result of vibration isolation by copper strings (see Fig. 5), the phase noise was found to be not affected by the vibrations of the cryocooler. This indicates, that cryocooler operation of whispering-gallery mode stabilised microwave oscillators is possible without performance degradation.

\section{ACKNOWLEDGMENT}

We like to thank Klaus Schieber from Bosch Telecom GmbH and Christian Neumann from Robert Bosch GmbH for designing and fabricating parts of the low-phase noise oscillator.

\section{REFERENCES}

11] V.B. Braginsky, V.S. Ilchenko, and Kh. S. Bagdassarov, "Experimental observation of fundamental microwave absorption in high quality dielectric crystals", Phys. Lett. A, vol. 120, pp. $300-305$, 1987.

[2] D.B. Leeson, "A simple model of feedback oscillator noise spectrum", Proc. IEEE, vol. 54, pp. 329-330, 1966.

[3] N.Klein, A.Scholen, N.Tellmann, C.Zuccaro and K.W.Urban, "Properties and applications of HTS-shielded dielectric resonators: a state-of-the-art report", IEEE Transactions on Microwave Theory and Techniques, vol. 44, pp. 1369-1373, 1996.

[4] N.Klein, C.Zuccaro, U.Dahne, H.Schulz, N.Tellmann, R.Kutzner, A.G.Zaitsev and R.Wordenweber, "Dielectric properties of rutile and its use in high temperature superconducting resonators", Journal of Applied Physics, vol. 78, pp. 6683-6686, 1995.

[5] M.Winter, N.Klein. L.Hao, and J.C.Gallop, "Cryogenic composite whispering-gallery mode resonators for low-phase noise frequency standards", IEEE Frequency Control Symposium 2000, Kansas City, to be published in the proceedings.

[6] D. Schmidt and T. Weiland, "Status and future of the $3 \mathrm{~d}$ mafia group of codes", IEEE Trans. Magn. vol. 28, pp. 1793-1796, 1992.

[7] L. Hao, N.Klein and J.C. Gallop, "Temperature compensated cryogenic whispering gallery mode resonator for microwave frequency standard applications", IEEE Transactions on Instrumentation and Measurement, vol. 48, pp. 524-527, 1999

[8] S.R. Stein and J.P. Turneaure, "The development of the superconducting cavity stabilized oscillator", Proc. IEEE vol. 63, 1249-1250, 1975.

[9] S. Chang, A.G.Mann, A.N. Luiten, "Cryogenic sapphire oscillator with improved frequency stability", IEEE Frequency Control Symposium 2000 , Kansas City, to be published in the proceedings 